\title{
Novel Ion Traps Using Planar Resistive Electrodes: Implications for Miniaturized Mass Analyzers
}

\author{
Daniel E. Austin,, ${ }^{a}$ Ying Peng, ${ }^{a}$ Brett J. Hansen, ${ }^{\mathrm{b}}$ Ivan W. Miller, ${ }^{\mathrm{a}}$ \\ Alan L. Rockwood, ${ }^{c}$ Aaron R. Hawkins, ${ }^{b}$ and Samuel E. Tolley ${ }^{\mathrm{d}}$ \\ ${ }^{a}$ Department of Chemistry and Biochemistry, Brigham Young University, Provo, Utah, USA \\ ${ }^{b}$ Department of Electrical and Computer Engineering, Brigham Young University, Provo, Utah, USA \\ ' Department of Pathology, University of Utah School of Medicine, and ARUP Laboratories, Salt Lake City, \\ Utah, USA \\ d Torion Technologies Inc., American Fork, Utah, USA
}

In radiofrequency ion traps, electric fields are produced by applying time-varying potentials between machined metal electrodes. The electrode shape constitutes a boundary condition and defines the field shape. This paper presents a new approach to making ion traps in which the electrodes consist of two ceramic discs, the facing surfaces of which are lithographically imprinted with sets of concentric metal rings and overlaid with a resistive material. A radial potential function can be applied to the resistive material such that the potential between the plates is quadrupolar, and ions are trapped between the plates. The electric field is independent of geometry and can be optimized electronically. The trap can produce any trapping field geometry, including both a toroidal trapping geometry and the traditional Paul-trap field. Dimensionally smaller ion trajectories, as would be produced in a miniaturized ion trap, can be achieved by increasing the potential gradient on the resistive material and operating the trap at higher frequency, rather than by making any physical changes to the trap or the electrodes. Obstacles to miniaturization of ion traps, such as fabrication tolerances, surface smoothness, electrode alignment, limited access for ionization or ion injection, and small trapping volume are addressed using this design. (J Am Soc Mass Spectrom 2008, 19, 1435-1441) (C 2008 American Society for Mass Spectrometry

I on traps are among the most widely used mass analyzers. Combining high sensitivity and chemical specificity, ion traps are used in applications ranging from proteomics to chemical warfare agent detection [1-4]. Ion traps are frequently used for tandem mass analysis and other ion dissociation techniques [5, 6]. Because ions can be accumulated and stored, ion molecule reactions can be carried out and studied within the trap [7-9]. Ion traps are frequently combined with gas chromatographs and other separation instruments [10, 11], enhancing their capabilities. The simple design and relatively high operating pressure make ion traps attractive choices for instrument miniaturization.

Several geometrical variations on the original quadrupole ion trap (Paul trap) have been developed, including cylindrical [12], rectilinear [13], linear [14], and toroidal [15] ion trap designs, each with advantages and disadvantages. For instance, rectilinear, linear, and toroidal traps have inherently larger storage volumes compared with quadrupole and cylindrical ion traps. In each of the trap geometries, ion trapping and mass

Address reprint requests to Dr. Daniel E. Austin, Department of Chemistry and Biochemistry, Brigham Young University, C-310 BNSN, Provo, UT 84602, USA. E-mail: austin@chem.byu.edu analysis is accomplished using radiofrequency quadrupolar potentials in at least two dimensions. In the Paul trap, three hyperboloidal electrodes trap ions in all three dimensions. In the rectilinear and linear traps, DC potentials on end plates trap ions in the third dimension. In the toroidal ion trap, the two-dimensional trapping field forms a closed loop.

In all ion trap variations, metal electrodes are used to produce the appropriate electric fields. For full-sized ion traps, modern machining equipment easily produces the hyperboloidal electrode surfaces of the quadrupole ion trap. For miniaturized traps, however, machining methods have been pushed to the limit, and simpler electrode geometries such as planar and cylindrical are required. For this reason, most miniaturized and microfabricated ion traps have utilized the cylindrical trap design [12, 16-20].

The need for a portable mass spectrometer has largely driven efforts to produce miniaturized ion traps [21]. Although the mass analyzer is just one of several components of a complete mass spectrometer system, miniaturization of the mass analyzer can often reduce the size and weight of other components. For example, the amplitude of ion motion is reduced in a small ion trap, so the mean free path of ions can be smaller (or 
more specifically, the number of collisions per rf period can remain the same with a higher collision frequency) $[21,22]$. As a result, miniature ion traps can be operated at higher pressure, reducing the size and weight of the vacuum pump. Another advantage is that smaller mass analyzers typically require less power, so the instrument can use smaller power supplies and lighter batteries.

Austin et al. [23] recently reported a new approach to making ion traps in which trapping fields are made using a combination of resistive material and metal electrode rings lithographically imprinted on ceramic disks. Potential functions superimposed on the resistive material produce trapping fields that resemble the fields created by shaped metal electrodes. This paper discusses the rationale and method for making ion traps using this approach, as well as a discussion and simulations of ion behavior in miniature traps.

\section{Theory}

\section{Nonequipotential Boundary Conditions}

Electric fields in ion traps can be determined by solving the Laplace equation $\left(\nabla^{2} \Phi=0\right)$ for a given set of boundary conditions. These boundary conditions are determined by the electrodes that form the trap. Metal electrodes produce equipotential boundary conditions, so the trapping fields are directly related to, and limited by, the geometry and arrangement of the electrodes.

Other approaches to produce quadrupolar trapping potentials have been explored. Wang and Wanczek proposed a method [24] in which several parallel ring electrodes in the shape of two abutting cones produce the same fields as a quadrupolar ion trap. The rf amplitude on the rings increases linearly from the smallest to the largest rings. This concept is suggestive of a more general approach, in which the electrode geometry can be independent of the trapping field geometry if the electrodes forming the trap can have an arbitrary potential function [25].

Consider the quadrupolar potential found in an ideal Paul-type ion trap. The time-independent potential along $\mathrm{x}, \mathrm{y}$, or $\mathrm{z}$ is quadratic everywhere in the trap:

$$
\Phi=A\left(x^{2}+y^{2}-2 z^{2}\right)+C
$$

in which the rotational axis of the trap lies in the $z$ direction. A plane parallel to the $x-y$ plane will have a quadratic potential function. Similarly, a cylindrical surface with a constant radius will have a potential that varies quadratically with $z$. If two such planes and this cylindrical surface form a closed volume, with quadratic potential functions on each surface, the fields within this volume will be identical to those made with hyperboloidal electrodes (Figure 1). By applying a radiofrequency signal, the time-dependent fields in this hypothetical volume will match the ideal Paul trap, and ion motion will be identical.

Surfaces with position-varying potential functions can be made using resistive material. However, if a voltage is applied at two points or edges of a resistive surface, the potential at each point is still constrained by the geometry of the material. For example, for a circular disk made out of resistive material with uniform thickness, a voltage applied between the center and outside edges creates a potential function that varies as $1 / \mathrm{r}$. Similarly, for a cylindrical tube of resistive material, a voltage applied between the two ends produces a linear potential function along the cylinder's length. This latter case is the basis for reflectrons and ion mobility drift tubes made from resistive glass [26, 27].

To create a useful electric field with the ability to control and optimize field shape, it is necessary to superimpose an arbitrary potential function on the resistive material. This can be accomplished using narrow electrode "wires" beneath the resistive material. For cylindrically-symmetric trapping fields, concentric electrode rings are used (Figure 2). The voltage on each ring is independently variable using a capacitive voltage divider or through other control electronics. Although the capacitive voltage divider and the resistive material both modify the voltage between each ring, the majority of the voltage dividing occurs as a result of the capacitors, not the resistive material. In other words, the resistive material is not the voltage divider. Similarly, very little current flows through the resistive material as the voltage across the capacitors oscillates. Thus, minimal heating of the resistive material occurs.

With a superimposed potential function, the resistive material performs several roles. The resistive material establishes a continuous boundary condition with a well-defined potential function, thereby creating the trapping fields. The resistive material prevents charge build-up from occurring, which would otherwise interfere with the fields of the trap. The superimposed

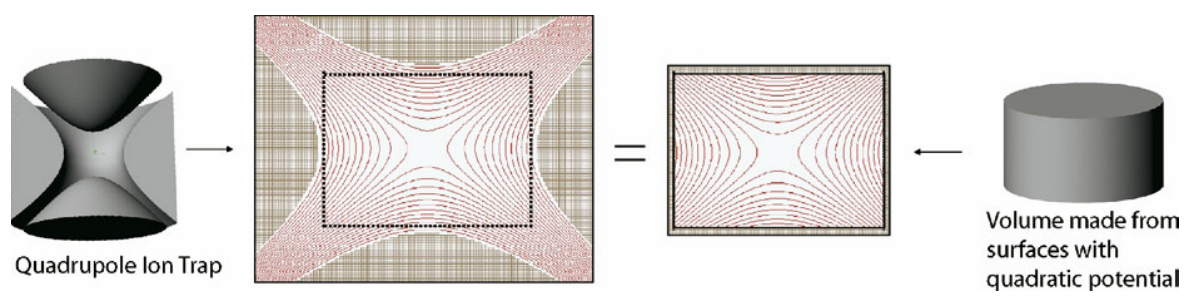

Figure 1. Quadratic potential functions on two planes and a cylinder produce a quadrupolar potential distribution identical to that in a trap made using hyperbolic metal electrodes. 

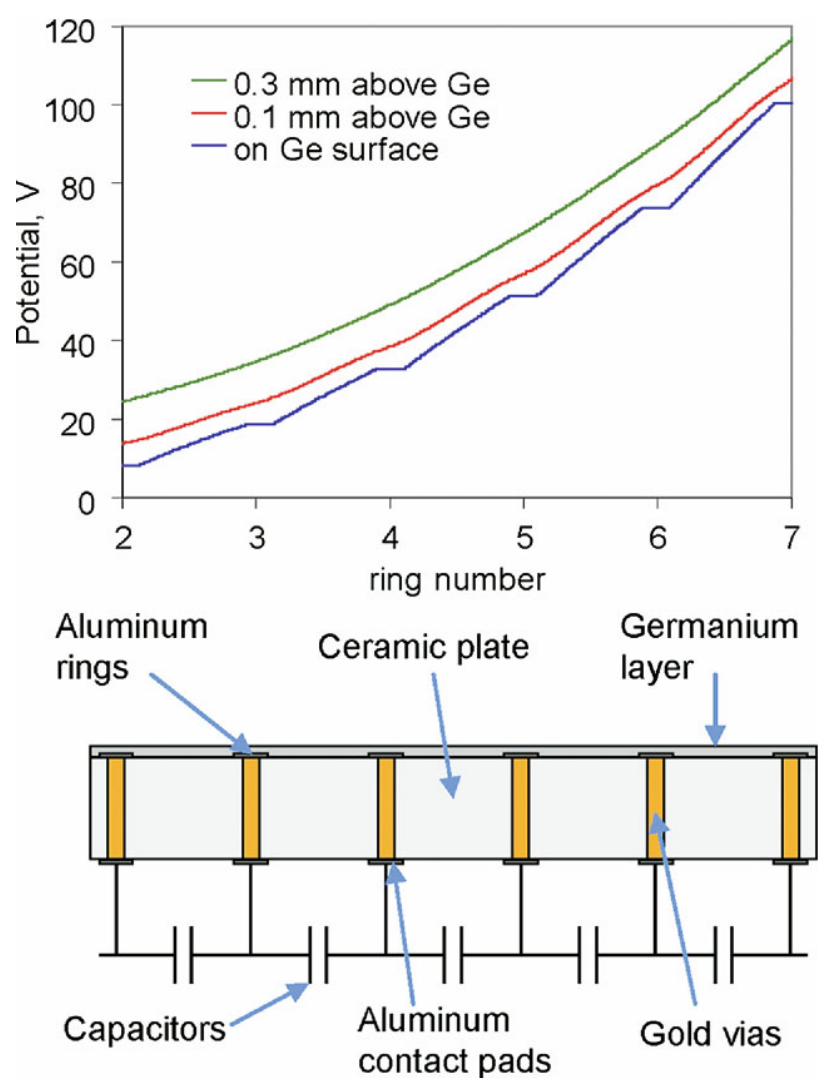

Figure 2. Electrode wires impose an arbitrary potential function on the overlaying resistive material. Plot shows the variation of the potential along the germanium surface and also 100 and $300 \mu$ above the germanium surface (within the trap).

potential function on the resistive material allows creation of electrical fields independent of the material geometry. As a result, the resistive material and electrode can be the simplest possible geometryplanar.

An example of this approach is the recentlyreported halo ion trap [23]. In the halo ion trap, resistive material (germanium) and underlying aluminum rings on the facing surfaces of two ceramic plates produce a toroidal trapping field. Trapped ions can be mass analyzed using resonance ejection. Ions are ejected through the center hole of one of the plates, and subsequently detected using an electron multiplier. A quadrupole ion trap, or Paul trap, can also be made using such plates. The same set of plates can produce both a toroidal trapping field and the traditional quadrupole trapping field, simply by changing the superimposed potential function on the resistive material. With machined metal electrodes, it is impossible to switch trapping field geometry in this manner.

\section{Ion Trap Miniaturization}

Several groups have reported miniaturized ion traps, almost exclusively of the cylindrical type [12, 16-20].
Ion trap miniaturization is constrained by the relationship of $m / z$ to the dimensional and operational parameters of the trap:

$$
\frac{m}{z}=\frac{8 V}{q_{z}\left(r_{0}^{2}+2 z_{0}^{2}\right) \Omega^{2}}
$$

where $q_{z}$ is the Mathieu stability parameter when the ion is ejected, $V$ and $\Omega$ are the amplitude and frequency of the rf applied to the trap, and $r_{0}$ and $z_{0}$ are the trap dimensions. Reducing $r_{0}$ and $z_{0}$ requires either increasing the trap frequency, reducing the rf amplitude, or both. Reducing the rf amplitude also reduces the pseudopotential well depth:

$$
\bar{D}_{z}=\frac{V q_{z}}{8} .
$$

As the well depth decreases, ion trapping and storage capacity decrease, so the rf amplitude cannot be arbitrarily small. On the other hand, as electrodes are placed closer together, the maximum voltage that can be sustained between them without electrical discharge goes down, again constraining the rf amplitude. The well depth and electrical discharge limit the range of voltage that can be used in voltage-ramp mass analysis. Some miniaturized traps get around this problem by using frequency scanning at constant $\mathrm{rf}$ amplitude.

One of the primary benefits of smaller traps derives from the decrease in the distance the ion travels per $\mathrm{rf}$ period. This deceased ion excursion allows the mean free path of ion-neutral collisions to be smaller, and hence the trap to operate at higher pressure with a smaller pump. However, the dimensions of ion excursion are not directly linked to the trap size. Rather, ion motion is governed by the magnitude of the trapping field and the trapping frequency.

The current paradigm of ion trap miniaturization is to make traps smaller. While this may seem obvious, it is not necessarily the only option. Any method that produces a higher trapping field and a higher trapping frequency will produce the same result as physical miniaturization of the trap electrodes. In the case of planar resistive electrodes, in which quadratic potential functions are imposed on the resistive material, as the plates are moved closer together, the trapping potentials remain quadrupolar. By increasing the field along the resistive material, the trapping field increases, reducing the amplitude of ion motion.

\section{Methods and Results}

Austin et al. [23] recently reported a new method for making ion traps in which trapping fields are made using two planar ceramic disks, the facing surfaces of which are imprinted with concentric metal rings, and overlaid with germanium. A radial potential function can be applied to the resistive material such that the 
field between the plates is quadrupolar, and ions are trapped between the plates. The metal rings impose an arbitrary potential function on the resistive material, which in turn establishes the field as seen by the ions. The shape of the trapping field is highly independent of the planar electrode shape, and in fact the same set of plates can be used to produce any geometry of trapping field, including quadrupole and toroidal ion traps.

Electrode plates are made using high-purity alumina $\left(\mathrm{Al}_{2} \mathrm{O}_{3}\right)$ wafers, $0.635 \mathrm{~mm}$ thick (Micro Substrate, Tempe, AZ). Holes are laser drilled for mounting, ion ejection, and electrical connections (vias) between the front and back sides of the plates. The vias are filled with gold, after which the plates are polished. Using standard photomask-photoresist techniques, aluminum electrodes are deposited on both sides of the plate: ring electrodes on the trapping side, leading to connection pads on the back side. Finally, a 50-nm layer of germanium is evaporated onto the trapping side of the plates. The resulting electrode plates are shown in Figure 3. Two plates are mounted together using a metal spacer.

The electric field between the plates is determined by the potentials put on each aluminum ring electrode. A capacitive voltage divider was used to produce the potentials for each ring. The resistance of the germanium layer contributes only a small amount to the voltage division.

Multiple independent ring potentials result in a multivariate solution for the time-independent potential at every point in the trap. In the present work, no effort has been made to find a rigorous solution to this system of equations. Rather, an initial guess for each ring potential, based on a quadratic potential function, was modified until a SIMION-generated plot of the trapping fields visually resembled those of traps made using curved metal electrodes.
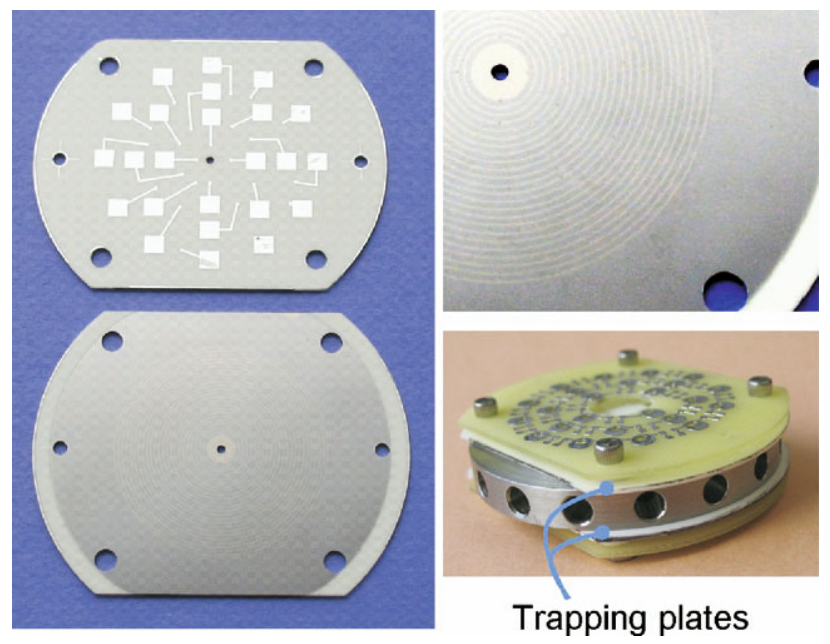

Figure 3. Planar resistive electrode plates used to produce a quadrupole ion trap. At left are shown top and back sides. Electrode rings are under the germanium and, therefore, cannot be seen easily, but can be seen in enlargement at upper right. Lower right shows trap assembly without voltage divider or connectors.
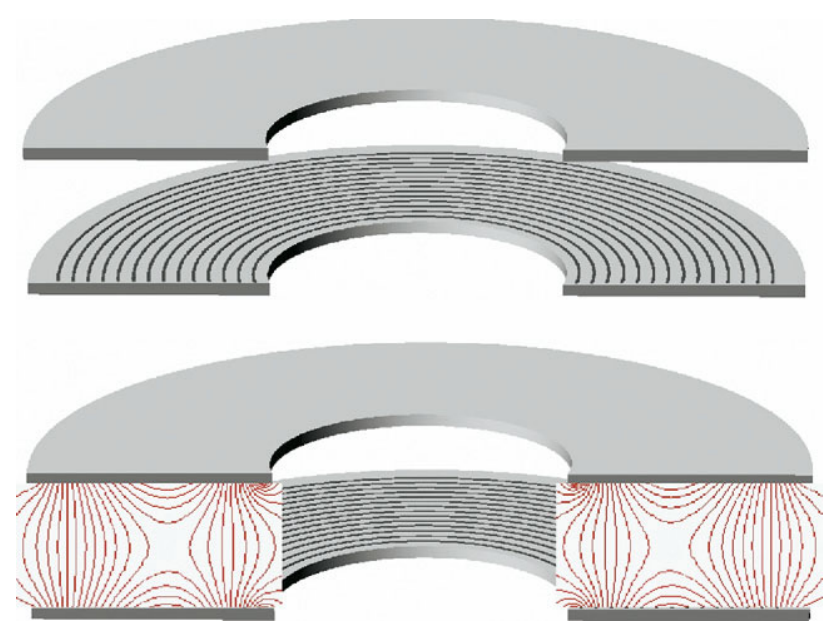

Figure 4. Toroidal trapping fields of the halo ion trap.

The potential function on the germanium surface (for instance, as shown in Figure 2) was modeled in SIMION using the following approach. First, ring electrodes were created as concentric cylindrical surfaces, and the potentials solved for the space between the cylindrical surfaces. Next, the points representing the space between the cylinders were converted to electrode points with the same potential they had as points in free space. After this, a radial plane of points was selected near the middle of the array, and all other points were converted to non-electrode points. At this point, the potential along this plane of points resembled the potential that would exist on a planar sheet of resistive material. Two such planes of points were used to determine the potentials within the trap. This method relies on the fact that the potential varies as $1 / \mathrm{r}$ in both a planar resistive material and in the space between cylindrical electrodes, and that the latter can be calculated quite well using the approximations of SIMION. Finally, the thickness of the underlying rings can be taken into account as the thickness of the cylindrical electrodes used in this approach.

In the previously-reported halo ion trap [23], planar resistive electrodes produced toroidal trapping fields (Figure 4), and ions were ejected to the center of the device using resonance ejection. Experiments using toluene, dichloromethane, and similar compounds showed mass resolution in the range of 50 to 100 $(m / \Delta m)$. Although the trapping fields in the vicinity of the center of the trapping volume agreed very well with the conventional toroidal ion trap, edge effects at the inside and outside of the plates (smaller and larger radial distances from rotational axis) produced significant field distortion that could not be completely eliminated by varying the ring potentials. Experiments in which a copper cylinder with a slit was placed inside this region did not improve resolution or sensitivity. Simulations using SIMION 7 [28] and SIMION 8 [29] show that the difficulty with this method is taking ions with primarily radial inward motion and pushing them 
out along one axis. Another difficulty is that the field drops off significantly near the center of the deviceagain an edge effect. Simulations of alternative mass analysis and ejection methods have been carried out. Simple mass-selective instability scanning, such as a frequency ramp or a voltage ramp, result in ions impacting the electrode plates, rather than ejecting in a radial direction. Efforts are currently underway to explore other options for ion ejection from the halo ion trap.

The same electrode plates have been used to produce an ion trap of the quadrupole (Paul trap) geometry. The only difference between this trap and the halo ion trap is the hole size at the center of the trap and the potential function imposed on the resistive material by the set of ring electrodes. Ions are trapped at the center of the device, in contrast to the halo trap. Ions are ejected axially. Simulations of a voltage ramp scan show much higher mass resolution than simulations of mass analysis in the halo trap.

\section{Simulations of Trap Miniaturization}

Miniaturization of metal-electrode ion traps requires making smaller electrodes. The boundary conditions must have the same shape to produce fields with the same shape. However, with planar resistive electrodes, the potential function on the electrode can be changed without changing the size of the physical electrode. An interesting result of this behavior is illustrated in Figure 5, which shows isopotential contours in the halo ion trap with plate spacing, $d$, of 4,2 , and $1 \mathrm{~mm}$. With a quadratic
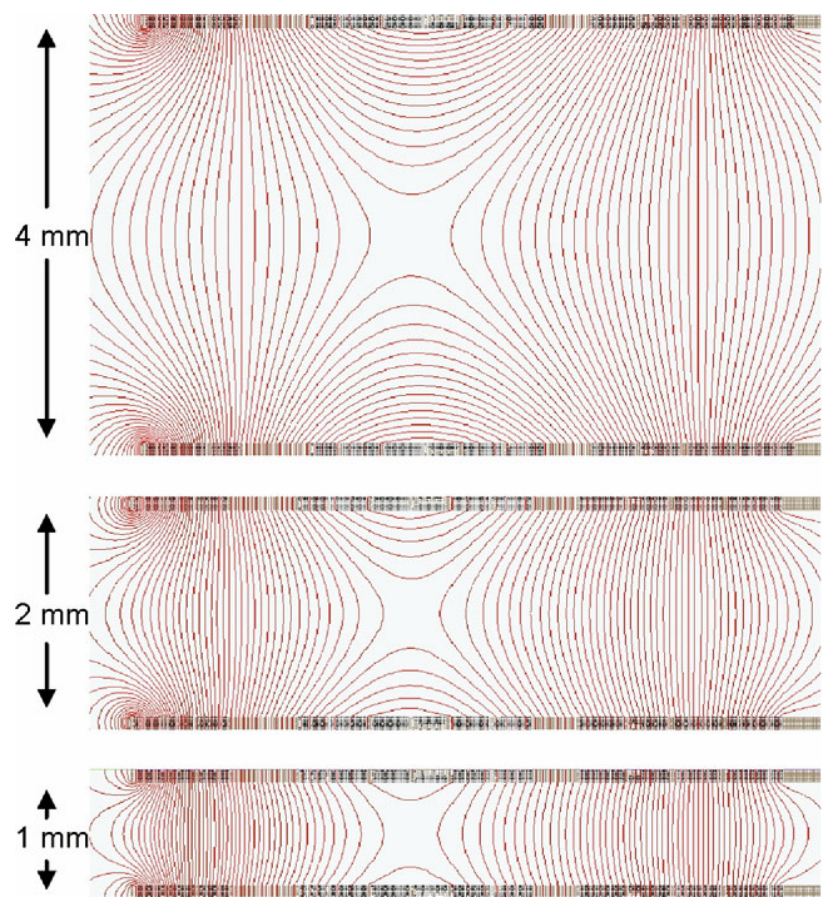

Figure 5. As plates are moved closer together, the trapping potential remains quadrupolar. The same potential function is used in each set of plates.
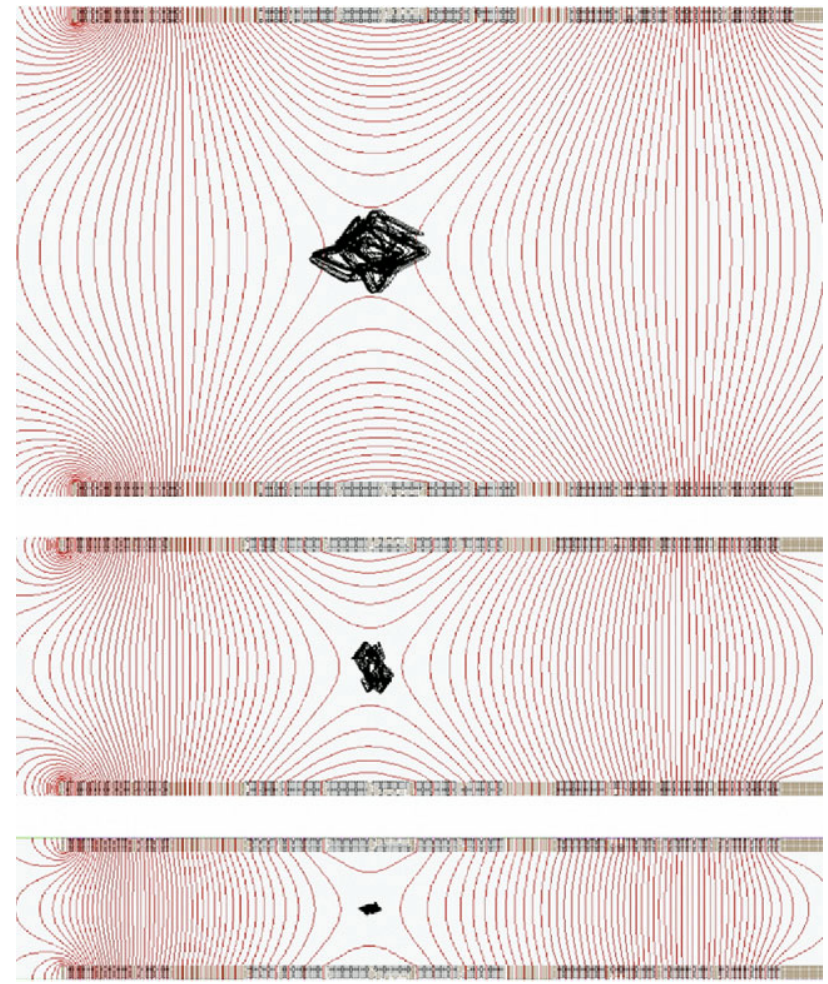

Figure 6. Simulations of ion motion as trapping plates are moved together. Plate separation is 4,2 , and $1 \mathrm{~mm}$.

potential function on both electrodes, the field between the electrodes remains quadrupolar regardless of the spacing between electrodes. The potential function on the plates in Figure 5 is identical for each set, and the resulting field shapes and magnitudes are identical (ignoring edge effects).

The dimensions of ion motion can be reduced in this trap by increasing the potential gradient applied between rings under the resistive material and simultaneously increasing the trapping frequency. Figure 6 shows SIMION 7 simulations of ion trajectories in traps under these conditions. The frequency in each case was chosen to provide a similar $\mathrm{q}_{\mathrm{z}}$ value for each set. Ions have $m / z=100 \mathrm{Th}$, and the ion trap is operated with $\mathrm{rf}$ amplitude of $600 \mathrm{~V}_{p-p}$. To facilitate an accurate comparison, ions originated at the same point in the rf phase, with $0.025 \mathrm{eV}$ kinetic energy. Ions experienced hardsphere collisions with helium in which the collision frequency was constrained to 0.05 the frequency of the driving rf. For plates separated by $4 \mathrm{~mm}$, the trapping frequency is $1.27 \mathrm{MHz}$, and the helium pressure is 0.8 mTorr. For each successively closer set of plates, the trapping frequency increases by a factor of 2 , and the helium pressure increases by roughly a factor of 2 . Ion excursion is reduced in each smaller scenario. When higher fields are created using the same plates, ion excursion and mean free path decrease, as they would in a miniaturized ion trap. In addition, the allowable pressure for trapping increases [21, 22]. Because fewer rings are used to create the field, the applied voltage on 
each plate is the same regardless of plate spacing. The distance over which this voltage is extended is reduced, resulting in an increased electric field at the trapping region.

As the plates are moved together, the radial pseudopotential well remains constant for a given voltage applied to each plate. Under these conditions, the pseudopotential well depth perpendicular to the plates is reduced. However, when the field is increased on the plates (by using fewer rings and the same applied voltage) the pseudopotential well depth perpendicular to the plates remains constant. Whereas the field in metal-electrode ion traps is limited by field emission between closely-spaced electrode surfaces, the field in resistive electrode ion traps is limited by the voltage difference between the closely-spaced ring electrodes. However, because of the large number of electrode rings, the voltage difference between adjacent electrodes is much smaller than the voltage difference between electrodes in conventional ion traps.

\section{Discussion}

It is important to note that changing the separation of the two planar resistive electrodes is not the same as shimming in conventional ion traps, nor does it directly modify the trapping field. In metal-electrode traps, changing the electrode separation changes the contribution of higher order multipoles in the trap. "Stretching" a trap increases the positive octopole contribution, compensating for electrode truncation and the ion entrance/exit holes, thereby improving ion ejection and mass resolution [29]. Several simulation studies [24, 30-34] have examined the effect of adding higher order multipoles in conventional traps. In planar resistive electrode traps, these higher order multipoles are added in by modifying the potential function on the resistive material. Changing the plate spacing leaves the field quadrupolar, with the same field magnitude, asymptote angles, etc., and modifies only the edge effects, with their corresponding higher order multipoles. Because higher order multipoles can be modified electronically, and are not constrained by physical geometry, new opportunities exist for producing and experimenting with modified fields. For instance, a nearly pure dipole can be produced and used for resonant ejection, whereas it is impossible to produce pure dipole fields using conventional ion traps. Finally, note the interesting result that the capacitance does not increase as the plates are moved together. For any point on one plate, the nearest point on the opposite plate has the exact same potential.

Surface roughness has been addressed as an issue facing miniaturized ion trap mass analyzers [35]. As traps get smaller, the effect of small surface deviations becomes larger relative to the trap size, and ion motion is consequently distorted. In the planar resistive electrode ion traps described herein, the surfaces producing the fields are polished to a surface roughness of better than $25 \mathrm{~nm}$ (root mean square), as measured by a surface profilometer. The uniformity of the thickness of the rings and germanium is estimated to be within 3-4 $\mathrm{nm}$. As a result, surface roughness and uniformity cease to be an issue for traps made using this method.

Similarly, electrode alignment becomes more critical for smaller ion traps. In microfabricated cylindrical ion trap arrays with $r_{0}=1$ and $5 \mu \mathrm{m}$, electrodes were visibly misaligned in STM images [30]. Misalignments of 10 to $40 \mu \mathrm{m}$ are tolerable for larger traps but hinder or prevent mass analysis in sub-mm ion traps. Planar resistive ion traps consist of only two pieces, making alignment simpler and more precise. Alignment of the two plates comprises five degrees of freedom (one of which has no effect on the trapping fields and can be ignored), whereas three-electrode traps (cylindrical, quadrupole) comprise 10 degrees of freedom, and rectilinear, linear, and toroidal traps comprise still more.

Closely related to both surface roughness and electrode alignment, machining tolerances become more important for smaller ion traps. In planar resistive ion traps, the largest deviation in the electrode structure comes from the tolerances of the photomask, specifically the position and uniform width of the ring electrodes. Typical tolerances in the current system are about 1 to $2 \mu \mathrm{m}$. Higher tolerances are achievable at increased cost.

Finally, miniaturized ion traps made using very small electrode elements can be mechanically fragile. In contrast, traps made using planar resistive electrodes do not have any free-standing structures. The ceramic plate can be made thicker for increased ruggedness without an effect on the trapping fields.

As ion traps are made smaller, the trapping volume and number of ions that can be analyzed is reduced. In addition, smaller traps have reduced access to the trapping region, making it harder to inject ions or to introduce electrons or photons into the trap. In the extreme case, simulations of ion trapping in micrometersized cylindrical ion traps show that only a single ion can remain trapped [36]. Trap arrays have been suggested as a solution to the reduced ion counts in smaller traps. In toroidal, linear, and rectilinear ion trapping geometries, ions are tightly confined in only two dimensions. The trapping volume extends along the third dimension, allowing more ions to be trapped and analyzed. For miniaturized systems, toroidal, linear, and rectilinear traps provide an alternative solution to reduced ion populations. A miniature toroidal, linear, or rectilinear ion trap made using planar resistive electrodes would combine the larger ion capacity of these trapping geometries with the larger access for ion injection available using planar electrodes. At the very small scale, arraying may still be needed, for which case traps made using planar electrodes are wellsuited. 


\section{Conclusions}

Miniaturized mass spectrometer systems will provide high analytical capabilities in a small package enabling numerous applications. Development of smaller mass analyzers is expected to allow size and weight reduction in the other instrument components, especially the vacuum system and power supplies. Many efforts at producing miniaturized and microfabricated ion trap mass analyzers have encountered several obstacles. Issues arise from mechanical tolerances, surface roughness, alignment of electrodes, shallower pseudopotential depth, limited applied voltage range, limited access to the trapping region, reduced ion trapping capacity, and difficulty of trap fabrication.

Although still in early development, ion traps made using planar resistive electrodes have several inherent advantages, many of which apply to miniaturization. Planar traps provide a simpler solution to mechanical tolerance, surface quality, fabrication, and alignment issues. Trap access is also improved. Trapping capacity can be increased by using a toroidal, linear, or rectilinear geometry. Further experiments are now being carried out to examine other opportunities and limitations of planar resistive electrode ion traps.

\section{Acknowledgments}

The authors acknowledge funding for this work by a grant from the NASA Planetary Instrument Definition and Development Program, NNH06ZDA001N.

\section{References}

1. Blanc, J. C. Y. L.; Hager, J. W.; Ilisiu, A. M. P.; Hunter, C.; Zhong, F.; Chu, I. Unique Scanning Capabilities of a New Hybrid Linear Ion Trap Mass Spectrometer (Q TRAP) Used for High Sensitivity Proteomics Applications. Proteomics 2003, 3, 859-869.

2. Xie, H.; Griffin, T. J. Trade-Off Between High Sensitivity and Increased Potential for False Positive Peptide Sequence Matches Using a TwoDimensional Linear Ion Trap for Tandem Mass Spectrometry-Based Proteomics. J. Proteome Res. 2006, 5, 1003-1009.

3. Laughlin, B. C.; Mulligan, C. C.; Cooks, R. G. Atmospheric Pressure Ionization in a Miniature Mass Spectrometer. Anal. Chem. 2005, 77, 2928-2939.

4. Riter, L. S.; Peng, Y. A.; Noll, R. J.; Patterson, G. E.; Aggerholm, T.; Cooks, R. G. Analytical Performance of a Miniature Cylindrical Ion Trap Mass Spectrometer. Anal. Chem. 2002, 74, 6154-6162.

5. Venable, J. D.; Wohlschlegel, J.; McClatchy, D. B.; Park, S. K.; Yates, J. R. Relative Quantification of Stable Isotope Labeled Peptides Using a Linear Ion Trap-Orbitrap Hybrid Mass Spectrometer. Anal. Chem. 2007, 79, 3056-3064.

6. Kosanam, H.; Prakash, P. K. S.; Yates, C. R.; Miller, D. D.; Ramagiri, S. Rapid Screening of Doping Agents in Human Urine by Vacuum MALDI-Linear Ion Trap Mass Spectrometry. Anal. Chem. 2007, 79, 6020-6026

7. Xia, Y.; Thomson, B. A.; McLuckey, S. A. Bidirectional Ion Transfer Between Quadrupole Arrays: $\mathrm{MS}^{n}$ Ion/Ion Reaction Experiments on a Quadrupole/Time-of-Flight Tandem Mass Spectrometer. Anal. Chem. 2007, 79, 8199-8206.

8. Liang, X. R.; McLuckey, S. A. Transmission Mode Ion/Ion Proton Transfer Reactions in a Linear Ion Trap. J. Am. Soc. Mass Spectrom. 2007, $18,882-890$

9. Sharifi, M.; Einhorn, J. Isomeric Differentiation of Conjugated Diene Epoxides by Polar $[4+2(+)]$ Diels-Alder Cycloaddition of Acylium Ions in an Ion Trap Mass Spectrometer. Int. J. Mass Spectrom. 1999, 191, 253-264.
10. Wang, Y.; Wu, S.-L.; Hancock, W. S. Monitoring of Glycoprotein Products in Cell Culture Lysates Using Lectin Affinity Chromatography and Capillary HPLC Coupled to Electrospray Linear Ion Trap-Fourier Transform Mass Spectrometry (LTQ/FTMS). Biotechnol. Prog. 2006, 22, 873-880.

11. Jin, W.-H.; Dai, J.; Li, S.-J.; Xia, Q.-C.; Zou, H.-F.; Zeng, R. Human Plasma Proteome Analysis by Multidimensional Chromatography Prefractionation and Linear Ion Trap Mass Spectrometry Identification. J. Proteome Res. 2005, 4, 613-619.

12. Cruz, D.; Chang, J. P.; Fico, M.; Guymon, A. J.; Austin, D. E.; Blain, M. G. Design, Microfabrication, and Analysis of Micrometer-Sized Cylindrical Ion Trap Arrays. Rev. Sci. Inst. 2007, 78, 015107-1-015107-9.

13. Ouyang, Z.; Wu, G.; Song, Y.; Li, H.; Plass, W. R.; Cooks, R. G. Rectilinear Ion Trap: Concepts, Calculations, and Analytical Performance of a New Mass Analyzer. Anal. Chem. 2004, 76, 4595-4605.

14. Douglas, D. J.; Frank, A. J.; Mao, D. Linear Ion Traps in Mass Spectrometry. Mass Spectrom. Rev. 2005, 24, 1-29.

15. Lammert, S. A.; Plass, W. R.; Thompson, C. V.; Wise, M. B. Design, Optimization and Initial Performance of a Toroidal rf Ion Trap Mass Spectrometer. Int. J. Mass Spectrom. 2001, 212, 25-40.

16. Van Amerom, A. F. H. W; Chaudhary, A.; Cardenas, M.; Bumgarner, J.; Short, R. T. Microfabrication of Cylindrical Ion Trap Mass Spectrometer Arrays for Handheld Chemical Analyzers. Chem. Eng. Commun. 2008, 195, 98-114.

17. Pau, S.; Pai, C. S.; Low, Y. L.; Moxom, J.; Reilly, P. T. A.; Whitten, W. B.; Ramsey, J. M. Microfabricated Quadrupole Ion Trap for Mass Spectrometer Applications. Phys. Rev. Lett. 2006, 96, 120801.

18. Badman, E. R.; Cooks, R. G. A Parallel Miniature Cylindrical Ion Trap Array. Anal. Chem. 2000, 72, 3291-3297.

19. Badman, E. R.; Cooks, R. G. Cylindrical Ion Trap Array with Mass Selection by Variation in Trap Dimensions. Anal. Chem. 2000, 72, 5079-5086.

20. Riter, L. S.; Meurer, E. C.; Handberg, E. S.; Laughlin, B. C.; Chen, H.; Patterson, G. E.; Eberlin, M. N.; Cooks, R. G. Ion/Molecule Reactions Performed in a Miniature Cylindrical Ion Trap Mass Spectrometer. Analyst 2003, 128, 1112-1118.

21. Badman, E. R.; Cooks, R. G. Special Feature: Perspective-Miniature Mass Analyzers. J. Mass Spectrom. 2000, 35, 659-671.

22. Ramsey, J. M.; Reilly, P. T. A.; Verbeck, G.; Whitten, W. B. Micro Ion Trap Mass Spectrometry. Proceedings of the 52nd ASMS Conference on Mass Spectrometry and Allied Topics; Nashville, TN, June, 2004.

23. Austin, D. E.; Wang, M.; Tolley, S. E.; Maas, J. D.; Hawkins, A. R. Rockwood, A. L.; Tolley, H. D.; Lee, E. D.; Lee, M. L. Halo Ion Trap Mass Spectrometer. Anal. Chem. 2007, 79, 2927-2932.

24. Wang, Y.; Wanczek, K. P. Generation of an Exact 3-Dimensioal Quadrupole Electron-Field and Superposition of a Homogeneous ElectricField and within a Common Closed Boundary with Application to Mass-Spectrometry. J. Chem. Phys. 1993, 98, 2647-2652.

25. Franzen, J.; Gabling, R.-H.; Schubert, M.; Wang, Y. In Practical Aspects of Ion Trap Mass Spectrometry, Vol. I. Fundamentals. March, R. E.; Todd, J. F. J.; Eds.; CRC Press: Boca Raton, FL, 1995, pp. 68-69.

26. Ritzau, S. M.; Laprade, B. N.; Mrotek, S. R.; Leffingwell, R. A Direct Comparison of a Resistive Glass and Stacked-Ring Reflectron. ASMS Abstract 2006, MP09 196

27. Laprade, B. N.; Mrotek, S.; Dunn, W.; Ritzau, S. The Development of Novel Resistive Glass Technology for Tailoring Electric Fields in Ion Mobility and Mass Spectrometer Applications. ASMS Abstract 2005, MP16 268.

28. Dahl, D. A. SIMION Version 7.0. Idaho National Engineering and Environmental Laboratory: Idaho Falls, ID, 2000.

29. Manura, D.; Dahl, D. A. SIMION Version 8.04. Scientific Instrument Services, Inc.: Ringoes, NJ, 2006.

30. Blain, M. G.; Riter, L. S.; Cruz, D.; Austin, D. E.; Wu, G. X.; Plass, W. R.; Cooks, R. G. Towards the Hand-Held Mass Spectrometer: Design Considerations, Simulation, and Fabrication of Micrometer-Scaled Cylindrical Ion Traps. Int. J. Mass Spectrom. 2004, 236, 91-104.

31. Tallapragada, P. K.; Mohanty, A. K.; Chatterjee, A.; Menon, A. G. Geometry Optimization of Axially Symmetric Ion Traps. Int. J. Mass Spectrom. 2007, 264, 38-52.

32. Chaudhary, A.; van Amerom, F. H. W.; Short, R. T.; Bhansali, S. Fabrication and Testing of a Miniature Cylindrical Ion Trap Mass Spectrometer Constructed from Low Temperature Cofired Ceramics. Int. J. Mass Spectrom. 2006, 251, 32-39.

33. Tabert, A. M.; Goodwin, M. P.; Cooks, R. G. Co-occurrence of Boundary and Resonance Ejection in a Multiplexed Rectilinear Ion Trap Mass Spectrometer. J. Am. Soc. Mass Spectrom. 2006, 17, 56-59.

34. Konenkov, N. V.; Cousins, L. M.; Baranov, V. I.; Sudakov, M. Y Quadrupole Mass Filter Operation with Auxiliary Quadrupolar Excitation: Theory and Experiment. Int. J. Mass Spectrom. 2001, 208, 17-27.

35. Xu, W.; Yu, M.; Ouyang, Z.; Cooks, G. R.; Chappell, W. Surface Roughness Effects on Dimensionally-Scaled Ion Traps. ASMS Abstract 2007, ТPB032

36. Austin, D. E.; Cruz, D.; Blain, M. G. Simulations of Ion Trapping Behavior in Micrometer-sized Cylindrical Ion Traps. J. Am. Soc. Mass Spectrom. 2006, 17, 430-441. 